\title{
ERROR BOUNDS OF GAUSSIAN QUADRATURE FORMULAE FOR ONE CLASS OF BERNSTEIN-SZEGÖ WEIGHTS
}

\author{
MIODRAG M. SPALEVIĆ
}

Abstract. The kernels $K_{n}(z)$ in the remainder terms $R_{n}(f)$ of the Gaussian quadrature formulae for analytic functions $f$ inside elliptical contours with foci at $\mp 1$ and a sum of semi-axes $\rho>1$, when the weight function $w$ is of Bernstein-Szegő type

$$
w(t) \equiv w_{\gamma}^{(-1 / 2,1 / 2)}(t)=\sqrt{\frac{1+t}{1-t}} \cdot \frac{1}{1-\frac{4 \gamma}{(1+\gamma)^{2}} t^{2}}, \quad t \in(-1,1), \quad \gamma \in(-1,0),
$$

are studied. Sufficient conditions are found ensuring that the kernel attains its maximal absolute value at the intersection point of the contour with the positive real semi-axis. This leads to effective error bounds of the corresponding Gauss quadratures. The quality of the derived bounds is analyzed by a comparison with other error bounds intended for the same class of integrands. In part our analysis is based on the well-known Cardano formulas, which are not very popular among mathematicians.

\section{INTRODUCTION}

Let the weight function $w$ be a nonnegative and integrable function on the interval $(-1,1)$. Consider the Gauss quadrature formula

$$
\int_{-1}^{1} f(t) w(t) d t=G_{n}[f]+R_{n}(f), \quad G_{n}[f]=\sum_{\nu=1}^{n} \lambda_{\nu} f\left(\tau_{\nu}\right) \quad(n \in \mathbb{N}),
$$

which is exact for all algebraic polynomials of degree at most $2 n-1$. The nodes $\tau_{\nu}$ in (1.1) are zeros of the orthogonal polynomials $\pi_{n}$ with respect to the weight function $w$.

In this paper $w$ is the weight function of Bernstein-Szegö type

$$
\begin{aligned}
w(t) & \equiv w_{\gamma}^{(-1 / 2,1 / 2)}(t) \\
& =\sqrt{\frac{1+t}{1-t}} \cdot\left(1-\frac{4 \gamma}{(1+\gamma)^{2}} t^{2}\right)^{-1}, \quad t \in(-1,1), \quad \gamma \in(-1,0) .
\end{aligned}
$$

Received by the editor September 22, 2010 and, in revised form, March 6, 2011.

2010 Mathematics Subject Classification. Primary 41A55; Secondary 65D30, 65D32.

Key words and phrases. Maximum, modulus, kernel, Gaussian quadrature formula, BernsteinSzegő weight function.

The author was supported in part by the Serbian Ministry of Education and Science (Research Project: "Methods of numerical and nonlinear analysis with applications", No. \#174002). 
The weight functions under consideration are special cases of the (more general) Bernstein-Szegő weight functions

$$
w_{\alpha, \beta, \delta}^{(-1 / 2,1 / 2)}(t)=\frac{\sqrt{\frac{1+t}{1-t}}}{\beta(\beta-2 \alpha) t^{2}+2 \delta(\beta-\alpha) t+\alpha^{2}+\delta^{2}}, \quad t \in(-1,1),
$$

where $0<\alpha<\beta, \beta \neq 2 \alpha,|\delta|<\beta-\alpha$, having in the denominator an arbitrary polynomial of exact degree 2 that remains positive on $[-1,1]$. Namely, if we set $\alpha=1, \beta=2 /(1+\gamma),-1<\gamma<0$, and $\delta=0$, (1.3) reduces to (1.2). The weight function (1.3) has been studied extensively in [1, and therefore the results obtained there can be specialized in the case of (1.2).

Let $\Gamma$ be a simple closed curve in the complex plane surrounding the interval $[-1,1]$ and $\mathcal{D}=\operatorname{int} \Gamma$ its interior. If the integrand $f$ is analytic in $\mathcal{D}$ and continuous on $\overline{\mathcal{D}}$, then the remainder term $R_{n}(f)$ in (1.1) admits the contour integral representation

$$
R_{n}(f)=\frac{1}{2 \pi i} \oint_{\Gamma} K_{n}(z) f(z) d z
$$

The kernel is given by

$$
K_{n}(z) \equiv K_{n}(z, w)=\frac{\varrho_{n}(z)}{\pi_{n}(z)}, \quad z \notin[-1,1],
$$

where

$$
\varrho_{n}(z) \equiv \varrho_{n, w}(z)=\int_{-1}^{1} \frac{\pi_{n}(t)}{z-t} w(t) d t .
$$

The modulus of the kernel is symmetric with respect to the real axis, i.e., $\left|K_{n}(\bar{z})\right|=\left|K_{n}(z)\right|$.

The integral representation (1.4) leads to the error estimate

$$
\left|R_{n}(f)\right| \leq \frac{\ell(\Gamma)}{2 \pi}\left(\max _{z \in \Gamma}\left|K_{n}(z)\right|\right)\left(\max _{z \in \Gamma}|f(z)|\right),
$$

where $\ell(\Gamma)$ is the length of the contour $\Gamma$. In order to get estimate (1.5), one has to study the magnitude of $\left|K_{n}(z)\right|$ on $\Gamma$.

In many papers error bounds of $\left|R_{n}(f)\right|$, where $f$ is an analytic function, are considered. Two choices of the contour $\Gamma$ have been widely used:

- a circle $C_{r}$ with a center at the origin and a radius $r(>1)$, i.e., $C_{r}=$ $\{z|| z \mid=r\}, r>1$ (cf. [2], [5, 6]), and

- an ellipse $\mathcal{E}_{\rho}$ with foci at the points $\mp 1$ and a sum of semi-axes $\rho>1$,

$$
\mathcal{E}_{\rho}=\left\{z \in \mathbb{C} \mid z=\frac{1}{2}\left(\rho e^{i \theta}+\rho^{-1} e^{-i \theta}\right), 0 \leq \theta \leq 2 \pi\right\} .
$$

When $\rho \rightarrow 1$ the ellipse shrinks to the interval $[-1,1]$, while with increasing $\rho$ it becomes more and more circle-like. The advantage of the elliptical contours, compared to the circular ones, is that such a choice needs the analyticity of $f$ in a smaller region of the complex plane, especially when $\rho$ is near 1 . In this paper we take $\Gamma$ to be the ellipse $\mathcal{E}_{\rho}$, then (1.5) has the form

$$
\left|R_{n}(f)\right| \leq \frac{\ell\left(\mathcal{E}_{\rho}\right)}{2 \pi}\left(\max _{z \in \mathcal{E}_{\rho}}\left|K_{n}(z)\right|\right)\left(\max _{z \in \mathcal{E}_{\rho}}|f(z)|\right) .
$$


Since the ellipse $\mathcal{E}_{\rho}$ has length $\ell\left(\mathcal{E}_{\rho}\right)=4 \varepsilon^{-1} E(\varepsilon)$, where $\varepsilon$ is the eccentricity of $\mathcal{E}_{\rho}$, i.e., $\varepsilon=2 /\left(\rho+\rho^{-1}\right)$, and $E(\varepsilon)=\int_{0}^{\pi / 2} \sqrt{1-\varepsilon^{2} \sin ^{2} \theta} d \theta$ is the complete elliptic integral of the second kind, the estimate (1.7) reduces to

$$
\left|R_{n}(f)\right| \leq \frac{2 E(\varepsilon)}{\pi \varepsilon}\left(\max _{z \in \mathcal{E}_{\rho}}\left|K_{n}(z)\right|\right)\|f\|_{\rho}, \quad \varepsilon=\frac{2}{\rho+\rho^{-1}},
$$

where $\|f\|_{\rho}=\max _{z \in \mathcal{E}_{\rho}}|f(z)|$. As we can see, the bound on the right-hand side in (1.8) is a function of $\rho$, so it can be optimized with respect to $\rho>1$.

The derivation of adequate bounds for $\left|R_{n}(f)\right|$ on the basis of (1.8) is possible only if good estimates for $\max _{z \in \mathcal{E}_{\rho}}\left|K_{n}(z)\right|$ are available. Especially useful is knowledge of the location of the extremal point $\eta \in \mathcal{E}_{\rho}$, at which $\left|K_{n}\right|$ attains its maximum. In such a case, instead of looking for upper bounds for $\max _{z \in \mathcal{E}_{\rho}}\left|K_{n}(z)\right|$, one can simply try to calculate $\left|K_{n}(\eta, w)\right|$. In general, this may not be an easy task, but in the case the Gauss-type quadrature formula (1.1) there exist effective algorithms for calculation of $K_{n}(z)$ at any point $z$ outside $[-1,1]$ (see Gautschi and Varga [2]). For a different approach to the estimation of $R_{n}(f)$, see [4].

So far the approach (1.8) was discussed for Gaussian quadrature rules (1.1) with respect to the Chebyshev weight functions (see [2], 3])

$$
w_{1}(t)=\frac{1}{\sqrt{1-t^{2}}}, \quad w_{2}(t)=\sqrt{1-t^{2}}, \quad w_{3}(t)=\sqrt{\frac{1+t}{1-t}}, \quad w_{4}(t)=\sqrt{\frac{1-t}{1+t}},
$$

and later was extended by Schira to symmetric weight functions under restriction of monotonicity type (either $w(t) \sqrt{1-t^{2}}$ is increasing on $(0,1)$ or $w(t) / \sqrt{1-t^{2}}$ is decreasing on $(0,1)$ ), including certain Gegenbauer weight functions (see [8]).

Recently (see 9]), with respect to the rational modification of the Chebyshev weight function of the second kind, i.e., the weight function of Bernstein-Szegö type

$$
w_{\gamma}^{(1 / 2)}(t)=\sqrt{1-t^{2}}\left(1-\frac{4 \gamma}{(1+\gamma)^{2}} t^{2}\right)^{-1}, \quad t \in(-1,1), \quad \gamma \in(-1,0),
$$

we found sufficient conditions ensuring that there exists a $\rho^{*}=\rho_{n}^{*}=\rho_{n, \gamma}^{*}$ such that for each $\rho \geq \rho^{*}$ the kernel $K_{n}$ attains its maximal absolute value at the intersection point of the ellipse with the imaginary axis. For this specialized case, we obtained much smaller values for $\rho=\rho_{n}^{*}$ than ones obtained by Schira (except for $\gamma$ close to 0 and $n$ even), especially for large values of $n$. Observe that the weight function $w_{\gamma}^{(1 / 2)}(t)$ belongs to the class considered by Schira [8] $\left(w_{\gamma}^{(1 / 2)}(t) / \sqrt{1-t^{2}}\right.$ is decreasing on $(0,1)$ ).

In [10] the same problem with respect to the weight function

$$
w_{\gamma}^{(-1 / 2)}(t)=\left(1 / \sqrt{1-t^{2}}\right) \cdot\left(1-\frac{4 \gamma}{(1+\gamma)^{2}} t^{2}\right)^{-1}, \quad t \in(-1,1), \quad \gamma \in(-1,0),
$$

has been considered. With respect to this symmetric weight function of BernsteinSzegő type, sufficient conditions are found ensuring that there exists a $\rho^{*}=\rho_{n}^{*}=$ $\rho_{n, \gamma}^{*}$ such that for each $\rho \geq \rho^{*}$ the kernel $K_{n}$ attains its maximal absolute value at the intersection point of the ellipse with either the real or the imaginary axis. The corresponding analysis is much more complicated than the one in 9]. Finally, observe that this weight function does not belong to the class of those considered by Schira 8 .

This paper is a continuation of the previous two [9, [10, as this methodology works well in the cases when the modulus of kernels have rather complicated forms. 
With respect to the weight function of Bernstein-Szego" type (1.2), which is not symmetric and therefore does not belong to the class of those considered by Schira [8], sufficient conditions are found ensuring that there exists a $\rho^{*}=\rho_{n}^{*}=\rho_{n, \gamma}^{*}$ such that for each $\rho \geq \rho^{*}$ the kernel $K_{n}$ attains its maximal absolute value at the intersection point of the ellipse with the positive real semi-axis. In an analogous way (using the substitution $t:=-t$ ) a similar analysis could be derived with respect to the weight function of Bernstein-Szegő type

$$
w_{\gamma}^{(1 / 2,-1 / 2)}(t)=\sqrt{\frac{1-t}{1+t}} \cdot\left(1-\frac{4 \gamma}{(1+\gamma)^{2}} t^{2}\right)^{-1}, \quad t \in(-1,1), \quad \gamma \in(-1,0),
$$

which is omitted.

Finally, let us note that in this paper we round off this problem with respect to the corresponding rational modification (of Bernstein-Szegö type) of all 4 classical Chebyshev weight functions.

\section{Maximum of the modulus of Kernels of Gaussian QuAdrature FORMULAE WITH THE WEIGHT FUNCTIONS $w_{\gamma}^{(-1 / 2,1 / 2)}$}

The corresponding (monic) orthogonal polynomial $\pi_{n}(t)$ of the degree $n$ has the form (see [5]):

$$
\begin{gathered}
\pi_{n}(t)=\pi_{n}^{(-1 / 2,1 / 2)}(t)=\frac{1}{2^{n}}\left[V_{n}(t)-\gamma V_{n-2}(t)\right], \quad n \geq 2, \\
\pi_{1}^{(-1 / 2,1 / 2)}(t)=t-(1+\gamma) / 2,
\end{gathered}
$$

where $V_{n}(n \geq 2)$ denotes the Chebyshev polynomial of the third kind.

As usual we use the substitution

$$
z=\frac{1}{2}\left(\xi+\xi^{-1}\right), \quad \xi=\rho e^{i \theta}
$$

Using the well-known facts (cf. [2])

$$
V_{n}(z)=\frac{\xi^{n+1}+\xi^{-n}}{\xi+1}, \quad z=\frac{1}{2}\left(\xi+\xi^{-1}\right),
$$

and

$$
\int_{-1}^{1} \frac{V_{n}(t)}{z-t} \sqrt{\frac{1+t}{1-t}} d t=\frac{2 \pi(\xi+1)}{\left(\xi-\xi^{-1}\right) \xi^{n+1}}
$$

on the basis of direct calculation, for $n \geq 2$ the kernel can be expressed in the following way:

$$
\begin{aligned}
& K_{n, \gamma}^{(-1 / 2,1 / 2)}(z)=\frac{\xi^{1 / 2}+\xi^{-1 / 2}}{\xi^{1 / 2}-\xi^{-1 / 2}} \\
& \quad \times \frac{2 \pi(1+\gamma)^{2}\left(1-\gamma \xi^{2}\right)}{\xi^{n+1 / 2}\left[(1+\gamma)^{2}-\gamma\left(\xi+\xi^{-1}\right)^{2}\right]\left[\left(\xi^{n+\frac{1}{2}}+\xi^{-n-\frac{1}{2}}\right)-\gamma\left(\xi^{n-\frac{3}{2}}+\xi^{-n+\frac{3}{2}}\right)\right]},
\end{aligned}
$$

and for $n=1$,

$$
K_{1, \gamma}^{(-1 / 2,1 / 2)}(z)=\frac{2 \pi(1+\gamma)^{2}\left(1-\gamma \xi^{2}\right)}{\xi^{3 / 2}\left(\xi+\xi^{-1}-1-\gamma\right)\left(\xi^{1 / 2}-\xi^{-1 / 2}\right)\left[(1+\gamma)^{2}-\gamma\left(\xi+\xi^{-1}\right)^{2}\right]} .
$$


From here on, we use the usual notation (see, for example, [2]):

$$
a_{j}=\frac{1}{2}\left(\rho^{j}+\rho^{-j}\right), \quad j \in \mathbb{N} .
$$

Using (2.3), and on the basis of

$$
\begin{gathered}
\left|1-\gamma \xi^{2}\right|=\left(1+\gamma^{2} \rho^{4}-2 \gamma \rho^{2} \cos 2 \theta\right)^{1 / 2} \\
\left|\xi^{k} \mp \xi^{-k}\right|=\sqrt{2}\left(a_{2 k} \mp \cos 2 k \theta\right)^{1 / 2} \\
\left|(1+\gamma)^{2}-\gamma\left(\xi+\xi^{-1}\right)^{2}\right|=\left[\left(1+\gamma^{2}\right)^{2}-4 \gamma\left(1+\gamma^{2}\right) a_{2} \cos 2 \theta\right. \\
\left.+2 \gamma^{2}\left(a_{4}+\cos 4 \theta\right)\right]^{1 / 2} \\
\left|\xi^{n+1 / 2}+\xi^{-n-1 / 2}-\gamma\left(\xi^{n-3 / 2}+\xi^{-n+3 / 2}\right)\right| \\
=\sqrt{2}\left[a_{2 n+1}+\cos (2 n+1) \theta+\gamma^{2}\left(a_{2 n-3}+\cos (2 n-3) \theta\right)\right. \\
\left.-2 \gamma\left(a_{2 n-1} \cos 2 \theta+a_{2} \cos (2 n-1) \theta\right)\right]^{1 / 2}
\end{gathered}
$$

we obtain (for $n \geq 2$ )

$$
\begin{aligned}
& \left|K_{n, \gamma}^{(-1 / 2,1 / 2)}(z)\right|=\left(\frac{a_{1}+\cos \theta}{a_{1}-\cos \theta}\right)^{1 / 2} \\
& \cdot \frac{\sqrt{2} \pi(1+\gamma)^{2}\left(1+\gamma^{2} \rho^{4}-2 \gamma \rho^{2} \cos 2 \theta\right)^{1 / 2}}{\rho^{n+1 / 2}\left[\left(1+\gamma^{2}\right)^{2}-4 \gamma\left(1+\gamma^{2}\right) a_{2} \cos 2 \theta+2 \gamma^{2}\left(a_{4}+\cos 4 \theta\right)\right]^{1 / 2}} \\
& \times \frac{1}{\left[a_{2 n+1}+\cos (2 n+1) \theta+\gamma^{2}\left(a_{2 n-3}+\cos (2 n-3) \theta\right)-2 \gamma\left(a_{2 n-1} \cos 2 \theta+a_{2} \cos (2 n-1) \theta\right)\right]^{1 / 2}} .
\end{aligned}
$$

Similarly, by using some of the formulas above and

$$
\left|\xi+\xi^{-1}-1-\gamma\right|=\left[2 a_{2}+(1+\gamma)^{2}+2 \cos 2 \theta-4(1+\gamma) a_{1} \cos \theta\right]^{1 / 2},
$$

for $n=1$ we have

$$
\begin{aligned}
& \left|K_{1, \gamma}^{(-1 / 2,1 / 2)}(z)\right| \\
& =\frac{\sqrt{2} \pi(1+\gamma)^{2}\left(1+\gamma^{2} \rho^{4}-2 \gamma \rho^{2} \cos 2 \theta\right)^{1 / 2}}{\rho^{3 / 2}\left(a_{1}-\cos \theta\right)^{1 / 2}\left[\left(1+\gamma^{2}\right)^{2}-4 \gamma\left(1+\gamma^{2}\right) a_{2} \cos 2 \theta+2 \gamma^{2}\left(a_{4}+\cos 4 \theta\right)\right]^{1 / 2}} \\
& \quad \times \frac{1}{\left[2 a_{2}+(1+\gamma)^{2}+2 \cos 2 \theta-4(1+\gamma) a_{1} \cos \theta\right]^{1 / 2}} .
\end{aligned}
$$

From here on, we analyze in details the much more important case $n \geq 2$, whereas the case $n=1$ will be mentioned in short.

Numerical results show that $\left|K_{n, \gamma}^{(-1 / 2,1 / 2)}(z)\right|, z \in \mathcal{E}_{\rho}$, attains its maximum on the positive real semi-axis for $\rho$ large enough $(n, \gamma$ are fixed). The graphs $\theta \mapsto$ $\left|K_{9,-0.99}^{(-1 / 2,1 / 2)}(z)\right|, z \in \mathcal{E}_{\rho}$, for $\rho=1.7$ (left) and $\rho=3.5$ (right), are displayed in Figure 1

Let $E, A, B, C, D ; E+E_{1}, A+A_{1}, B+B_{1}, C+C_{1}, D+D_{1}$ be positive real terms. Further on we will use the fact that

$$
\frac{E+E_{1}}{B+B_{1}} \cdot \frac{A+A_{1}}{\left(C+C_{1}\right)\left(D+D_{1}\right)} \leq \frac{E A}{B C D},
$$



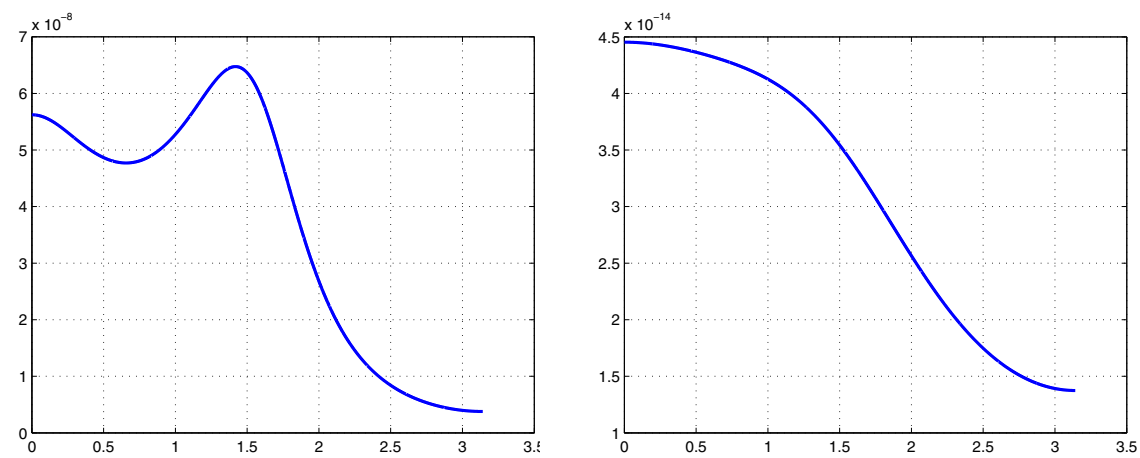

Figure 1. The graphs $\theta \mapsto\left|K_{9,-0.99}^{(-1 / 2,1 / 2)}(z)\right|, z=r(\theta) e^{i \theta} \in \mathcal{E}_{\rho}$, for $\rho=1.7$ (left) and $\rho=3.5$ (right).

is equivalent to

$$
\begin{aligned}
& E A\left(B+B_{1}\right) C_{1}\left(D+D_{1}\right)+E A C\left(B+B_{1}\right) D_{1}+A C D\left(E B_{1}-B E_{1}\right) \\
& \quad-B C D A_{1}\left(E+E_{1}\right) \geq 0 .
\end{aligned}
$$

Theorem 2.1. For the Gauss quadrature formula (1.1) with the weight function (1.2) there exists $\rho^{*}=\rho_{n}^{*}=\rho_{n, \gamma}^{*} \in(1,+\infty)$ such that for each $\rho>\rho^{*}$ the modulus of the kernel $\left|K_{n, \gamma}^{(-1 / 2,1 / 2)}(z)\right|$ attains its maximum on the real axis (positive real semi-axis $(\theta=0))$, i.e.,

$$
\max _{z \in \mathcal{E}_{\rho}}\left|K_{n, \gamma}^{(-1 / 2,1 / 2)}(z)\right|=K_{n, \gamma}^{(-1 / 2,1 / 2)}\left(\frac{1}{2}\left(\rho+\rho^{-1}\right)\right),
$$

for $\gamma \in(-1,0)$.

Proof. The assertion of the theorem holds if $(2.8)$

$$
\begin{aligned}
& \frac{a_{1}+\cos \theta}{a_{1}-\cos \theta} \cdot \frac{1+\gamma^{2} \rho^{4}-2 \gamma \rho^{2} \cos 2 \theta}{\left(1+\gamma^{2}\right)^{2}-4 \gamma\left(1+\gamma^{2}\right) a_{2} \cos 2 \theta+2 \gamma^{2}\left(a_{4}+\cos 4 \theta\right)} \\
& \times \frac{1}{a_{2 n+1}+\cos (2 n+1) \theta+\gamma^{2}\left(a_{2 n-3}+\cos (2 n-3) \theta\right)-2 \gamma\left(a_{2 n-1} \cos 2 \theta+a_{2} \cos (2 n-1) \theta\right)} \\
& \leq \frac{a_{1}+1}{a_{1}-1} \cdot \frac{1+\gamma^{2} \rho^{4}-2 \gamma \rho^{2}}{\left(1+\gamma^{2}\right)^{2}-4 \gamma\left(1+\gamma^{2}\right) a_{2}+2 \gamma^{2}\left(a_{4}+1\right)} \\
& \times \frac{1}{a_{2 n+1}+1+\gamma^{2}\left(a_{2 n-3}+1\right)-2 \gamma\left(a_{2 n-1}+a_{2}\right)}
\end{aligned}
$$

holds for each $\rho>\rho^{*}$ and for $\theta \in[0, \pi]$. 
According to (2.6) and (2.7) with

$$
\begin{aligned}
E= & a_{1}+1(\geq 0), \quad E_{1}=-2 \sin ^{2} \frac{\theta}{2}, \\
A= & 1+\gamma^{2} \rho^{4}-2 \gamma \rho^{2}(\geq 0), \quad A_{1}=4 \gamma \rho^{2} \sin ^{2} \theta, \\
B= & a_{1}-1(\geq 0), \quad B_{1}=2 \sin ^{2} \frac{\theta}{2} \\
C= & \left(1+\gamma^{2}\right)^{2}-4 \gamma\left(1+\gamma^{2}\right) a_{2}+2 \gamma^{2}\left(a_{4}+1\right)(\geq 0), \\
C_{1}= & 8 \gamma\left(1+\gamma^{2}\right) a_{2} \sin ^{2} \theta-4 \gamma^{2} \sin ^{2} 2 \theta \\
D= & a_{2 n+1}+1+\gamma^{2}\left(a_{2 n-3}+1\right)-2 \gamma\left(a_{2 n-1}+a_{2}\right)(\geq 0), \\
D_{1}= & -2 \sin ^{2} \frac{(2 n+1) \theta}{2}-2 \gamma^{2} \sin ^{2} \frac{(2 n-3) \theta}{2} \\
& +4 \gamma a_{2 n-1} \sin ^{2} \theta+4 \gamma a_{2} \sin ^{2} \frac{(2 n-1) \theta}{2},
\end{aligned}
$$

(2.8) reduces to the inequality which holds trivially for $\theta=0$ and $\theta=\pi$, and for other values of $\theta$, one may divide by $2 \sin ^{2}(\theta / 2) \cos ^{2}(\theta / 2)(\theta \in(0, \pi))$ to obtain $(2.9)$

$$
\begin{aligned}
& 2\left(a_{1}+1\right)\left(1+\gamma^{2} \rho^{4}-2 \gamma \rho^{2}\right)\left(a_{1}-\cos \theta\right)\left(8 \gamma\left(1+\gamma^{2}\right) a_{2}-16 \gamma^{2} \cos ^{2} \theta\right) \\
& \times\left(a_{2 n+1}+\cos (2 n+1) \theta+\gamma^{2}\left(a_{2 n-3}+\cos (2 n-3) \theta\right)\right. \\
& \left.\quad-2 \gamma\left(a_{2 n-1} \cos 2 \theta+a_{2} \cos (2 n-1) \theta\right)\right) \\
& +8 \gamma a_{2 n-1}\left(a_{1}+1\right)\left(1+\gamma^{2} \rho^{4}-2 \gamma \rho^{2}\right)\left(\left(1+\gamma^{2}\right)^{2}\right. \\
& \left.\quad-4 \gamma\left(1+\gamma^{2}\right) a_{2}+2 \gamma^{2}\left(a_{4}+1\right)\right)\left(a_{1}-\cos \theta\right) \\
& +\frac{1}{\cos ^{2}(\theta / 2)}\left(1+\gamma^{2} \rho^{4}-2 \gamma \rho^{2}\right)\left(\left(1+\gamma^{2}\right)^{2}-4 \gamma\left(1+\gamma^{2}\right) a_{2}+2 \gamma^{2}\left(a_{4}+1\right)\right) \\
& \times\left[2 a_{1}\left(a_{2 n+1}+1+\gamma^{2}\left(a_{2 n-3}+1\right)-2 \gamma\left(a_{2 n-1}+a_{2}\right)\right)-\left(a_{1}+1\right)\left(a_{1}-\cos \theta\right)\right. \\
& \left.\times\left(\frac{\sin ^{2} \frac{(2 n+1) \theta}{2}}{\sin ^{2} \frac{\theta}{2}}+\gamma^{2} \frac{\sin ^{2} \frac{(2 n-3) \theta}{2}}{\sin ^{2} \frac{\theta}{2}}-2 \gamma a_{2} \frac{\left.\sin ^{2} \frac{(2 n-1) \theta}{2}\right)}{\sin ^{2} \frac{\theta}{2}}\right)\right] \\
& -8 \gamma \rho^{2}\left(a_{1}-1\right)\left(\left(1+\gamma^{2}\right)^{2}-4 \gamma\left(1+\gamma^{2}\right) a_{2}+2 \gamma^{2}\left(a_{4}+1\right)\right) \\
& \times\left(a_{2 n+1}+1+\gamma^{2}\left(a_{2 n-3}+1\right)-2 \gamma\left(a_{2 n-1}+a_{2}\right)\right)\left(a_{1}+\cos \theta\right) \geq 0 .
\end{aligned}
$$

Observe that, since $a_{1}-\cos \theta \leq a_{1}+1$,

$$
\begin{gathered}
\frac{\sin ^{2} \frac{\ell \theta}{2}}{\sin ^{2} \frac{\theta}{2}} \leq \ell^{2}, \quad \ell=2 n+1,2 n-3,2 n-1, \\
2 a_{1}\left(a_{2 n+1}+1+\gamma^{2}\left(a_{2 n-3}+1\right)-2 \gamma\left(a_{2 n-1}+a_{2}\right)\right)-\left(a_{1}+1\right)\left(a_{1}-\cos \theta\right) \\
\times\left(\frac{\sin ^{2} \frac{(2 n+1) \theta}{2}}{\sin ^{2} \frac{\theta}{2}}+\gamma^{2} \frac{\sin ^{2} \frac{(2 n-3) \theta}{2}}{\sin ^{2} \frac{\theta}{2}}-2 \gamma a_{2} \frac{\sin ^{2} \frac{(2 n-1) \theta}{2}}{\sin ^{2} \frac{\theta}{2}}\right) \\
\geq 2 a_{1}\left(a_{2 n+1}+1+\gamma^{2}\left(a_{2 n-3}+1\right)-2 \gamma\left(a_{2 n-1}+a_{2}\right)\right)-\left(a_{1}+1\right)^{2} \\
\times\left((2 n+1)^{2}+\gamma^{2}(2 n-3)^{2}-2 \gamma a_{2}(2 n-1)^{2}\right) .
\end{gathered}
$$


There exists $\rho_{0}(>1)$ such that the expression on the right-hand side of the sign $\geq$ in the last inequality is greater than or equal to 0 for each $\rho>\rho_{0}$, since it is equal to $\frac{1}{2} \rho^{2 n+2}+O\left(\rho^{2 n}\right), \rho \rightarrow \infty$. Therefore, for each $\rho>\rho_{0}$ it holds that

$$
\begin{aligned}
& \frac{1}{\cos ^{2}(\theta / 2)} \cdot\left[2 a_{1}\left(a_{2 n+1}+1+\gamma^{2}\left(a_{2 n-3}+1\right)-2 \gamma\left(a_{2 n-1}+a_{2}\right)\right)-\left(a_{1}+1\right)\left(a_{1}-\cos \theta\right)\right. \\
& \left.\times\left(\frac{\sin ^{2} \frac{(2 n+1) \theta}{2}}{\sin ^{2} \frac{\theta}{2}}+\gamma^{2} \frac{\sin ^{2} \frac{(2 n-3) \theta}{2}}{\sin ^{2} \frac{\theta}{2}}-2 \gamma a_{2} \frac{\sin ^{2} \frac{(2 n-1) \theta}{2}}{\sin ^{2} \frac{\theta}{2}}\right)\right] \\
& \geq 2 a_{1}\left(a_{2 n+1}+1+\gamma^{2}\left(a_{2 n-3}+1\right)-2 \gamma\left(a_{2 n-1}+a_{2}\right)\right)-\left(a_{1}+1\right)^{2} \\
& \times\left((2 n+1)^{2}+\gamma^{2}(2 n-3)^{2}-2 \gamma a_{2}(2 n-1)^{2}\right) .
\end{aligned}
$$

Now, we conclude that (2.9) holds, if

$$
\begin{aligned}
& 2\left(a_{1}+1\right)^{2}\left(1+\gamma^{2} \rho^{4}-2 \gamma \rho^{2}\right)\left(8 \gamma\left(1+\gamma^{2}\right) a_{2}-16 \gamma^{2}\right) \\
& \times\left(a_{2 n+1}+1+\gamma^{2}\left(a_{2 n-3}+1\right)-2 \gamma\left(a_{2 n-1}+a_{2}\right)\right) \\
& +8 \gamma a_{2 n-1}\left(a_{1}^{2}-1\right)\left(1+\gamma^{2} \rho^{4}-2 \gamma \rho^{2}\right)\left(\left(1+\gamma^{2}\right)^{2}-4 \gamma\left(1+\gamma^{2}\right) a_{2}+2 \gamma^{2}\left(a_{4}+1\right)\right) \\
& +\left(1+\gamma^{2} \rho^{4}-2 \gamma \rho^{2}\right)\left(\left(1+\gamma^{2}\right)^{2}-4 \gamma\left(1+\gamma^{2}\right) a_{2}+2 \gamma^{2}\left(a_{4}+1\right)\right) \\
& \times\left[2 a_{1}\left(a_{2 n+1}+1+\gamma^{2}\left(a_{2 n-3}+1\right)-2 \gamma\left(a_{2 n-1}+a_{2}\right)\right)-\left(a_{1}+1\right)^{2}\right. \\
& \left.\times\left((2 n+1)^{2}+\gamma^{2}(2 n-3)^{2}-2 \gamma a_{2}(2 n-1)^{2}\right)\right] \\
& -8 \gamma \rho^{2}\left(a_{1}-1\right)^{2}\left(\left(1+\gamma^{2}\right)^{2}-4 \gamma\left(1+\gamma^{2}\right) a_{2}+2 \gamma^{2}\left(a_{4}+1\right)\right) \\
& \times\left(a_{2 n+1}+1+\gamma^{2}\left(a_{2 n-3}+1\right)-2 \gamma\left(a_{2 n-1}+a_{2}\right)\right) \geq 0 .
\end{aligned}
$$

Therefore, there exists $\rho^{*}\left(>\rho_{0}\right)$ such that the expression on the right-hand side of the sign $\geq$ in the last inequality is greater than or equal to 0 for each $\rho>\rho^{*}$, since it is equal to $\frac{1}{2} \gamma^{4} \rho^{2 n+10}+O\left(\rho^{2 n+9}\right), \rho \rightarrow \infty$. The conclusion follows.

From the practical point of view, in the Appendix we present effective methods in finding intervals $\left[\rho^{*}, \infty\right)$ so that $\rho^{*}$ is as small as possible, and so that the kernel $K_{n, \gamma}^{(-1 / 2,1 / 2)}$ attains its maximum absolute value at the intersection point of the ellipse $\mathcal{E}_{\rho}\left(\rho \in\left[\rho^{*}, \infty\right)\right)$ with the positive real semi-axis.

In the case that $n=1$ holds, the following theorem, whose proof is similar to that of Theorem 2.1. will be omitted.

Theorem 2.2. If $n=1$, for the Gauss quadrature formula (1.1) with the weight function (1.2) there exists a $\rho_{1}^{*}=\rho_{1, \gamma}^{*} \in(1,+\infty)$ such that for each $\rho>\rho_{1}^{*}$ the modulus of the kernel $\left|K_{1, \gamma}^{(-1 / 2,1 / 2)}(z)\right|$ attains its maximum on the real axis (positive real semi-axis $(\theta=0))$, i.e.,

$$
\max _{z \in \mathcal{E}_{\rho}}\left|K_{1, \gamma}^{(-1 / 2,1 / 2)}(z)\right|=K_{1, \gamma}^{(-1 / 2,1 / 2)}\left(\frac{1}{2}\left(\rho+\rho^{-1}\right)\right),
$$

for $\gamma \in(-1,0)$.

\section{NuMERICAL RESULTS}

The values of $\rho^{*}=\rho_{n}^{*}$ which appear in Theorem 2.1 depend on $\gamma$, i.e., $\rho_{n}^{*}=$ $\rho_{n}^{*}(\gamma)$. The results obtained by our method show that the values $\rho_{n}^{*}=\rho_{n}^{*}(\gamma)(>1)$, $\gamma \in(-1,0)$, become very close to 1 for large values of $n$ and $\gamma$ close to 0 , and should 
provide the very effective error bounds of type (1.7). The disadvantage of the error bounds of type (1.7) might appear in the case when $\rho_{n}^{*}=\rho_{n}^{*}(\gamma)$ is not close to 1 .

Analogously, as in [9], 10], let us consider numerical calculation of the integral

$$
I(f)=\int_{-1}^{1} f(t) \sqrt{\frac{1+t}{1-t}} \cdot\left(1-\frac{4 \gamma}{(1+\gamma)^{2}} t^{2}\right)^{-1} d t .
$$

Under the assumption that $f$ is analytic inside $\mathcal{E}_{\rho_{\max }}$, from (1.7) we obtain the error bound

$$
\left|R_{n}(f)\right| \leq \tilde{e}_{n}(f)
$$

where

$$
\tilde{e}_{n}(f)=\inf _{\rho_{n}^{*}<\rho<\rho_{\max }}\left[\frac{\ell\left(\mathcal{E}_{\rho}\right)}{2 \pi}\left(\max _{z \in \mathcal{E}_{\rho}}\left|K_{n}(z)\right|\right)\left(\max _{z \in \mathcal{E}_{\rho}}|f(z)|\right)\right],
$$

and $\rho_{n}^{*}$ is defined by Theorem 2.1

The length of the ellipse $\mathcal{E}_{\rho}$ is estimated by (see [7, Eq. (2.2)])

$$
\ell\left(\mathcal{E}_{\rho}\right) \leq 2 \pi a_{1}\left(1-\frac{1}{4} a_{1}^{-2}-\frac{3}{64} a_{1}^{-4}-\frac{5}{256} a_{1}^{-6}\right),
$$

where $a_{1}=\left(\rho+\rho^{-1}\right) / 2$.

Let the integrand $f$ in (3.1) be $f(t)=e^{e^{t}} \cdot\left[(a+t)^{k}(b+t)^{\ell}(c+t)^{m}\right]^{-1}$, where $a<$ $-1, c \leq b \leq a ; k \in \mathbb{N}, \ell, m \in \mathbb{N}_{0}$. The condition $a<-1, c \leq b \leq a$ means that the function $f$ is analytic inside the elliptical contour $\mathcal{E}_{\rho_{\max }}$, where $|\bar{a}|=\frac{1}{2}\left(\rho_{\max }+\rho_{\max }^{-1}\right)$. The classical error bound in this case is difficult to determine, since the derivatives $f^{(2 n)}(t)$ for higher values of $n$ are too complicated to be handled. However, we can use the error bound (3.2) based on the results of Theorem 2.1, if $\rho^{*}<\rho_{\max }$.

One can prove that (see [9])

$$
\max _{z \in \mathcal{E}_{\rho}}\left|\frac{e^{e^{z}}}{(a+z)^{k}(b+z)^{\ell}(c+z)^{m}}\right|=\frac{e^{e^{a_{1}}}}{\left|a+a_{1}\right|^{k}\left|b+a_{1}\right|^{\ell}\left|c+a_{1}\right|^{m}},
$$

where the maximum is attained at $\theta=0$.

On the basis of the obtained results, $\tilde{e}_{n}(f)$, in the case $\gamma \in(-1,0), n \geq 2$, can be bounded by

$$
\begin{aligned}
\tilde{e}_{n}(f) \leq e_{n}(f) & =\inf _{\rho_{n}^{*}<\rho<\rho_{\max }}\left\{\sqrt{2} \pi a_{1}\left(1-\frac{1}{4} a_{1}^{-2}-\frac{3}{4} a_{1}^{-4}-\frac{5}{256} a_{1}^{-6}\right)\right. \\
& \times e^{e^{a_{1}}} \cdot\left[\left|a+a_{1}\right|^{k}\left|b+a_{1}\right|^{\ell}\left|c+a_{1}\right|^{m}\right]^{-1} \\
& \times \frac{(1+\gamma)^{2}\left(a_{1}+1\right)^{1 / 2}\left(1-\gamma \rho^{2}\right)}{\rho^{n+1 / 2}\left(a_{1}-1\right)^{1 / 2}\left[\left(1+\gamma^{2}\right)^{2}-4 \gamma\left(1+\gamma^{2}\right) a_{2}+2 \gamma^{2}\left(a_{4}+1\right)\right]^{1 / 2}} \\
& \left.\times\left[a_{2 n+1}+1+\gamma^{2}\left(a_{2 n-3}+1\right)-2 \gamma\left(a_{2 n-1}+a_{2}\right)\right]^{-1 / 2}\right\} .
\end{aligned}
$$

The error bound (3.2) is valid for integrands analytic in a neighborhood of the interval of integration and should be compared with other error bounds intended for the same class of integrands. There are several classical error bounds for Gaussian quadrature rules of analytic functions. See Theorem 4 in [11] or Theorem 1 in [12], where the contour $\Gamma$ is the ellipse $\mathcal{E}_{\rho}$ given by (1.6). We also take into account the error bounds appearing in [5], where the contour $\Gamma$ is the circumference $C_{r}=\{z \in$ $\mathbb{C}:|z|=r\}(r>1)$. 
Therefore, the error bound $\hat{e}_{n}(f)\left(\left|R_{n}(f)\right| \leq \hat{e}_{n}(f)\right)$ of the Gauss quadrature formula (1.1) with respect to the weight function (1.2), for the integrand $f$ under consideration, can be given by (see Stenger [11, Eq. (38)])

$$
\hat{e}_{n}(f)=\hat{e}_{n}^{(\text {Sten })}(f)=\inf _{1<\rho<\rho_{\max }}\left\{\frac{16 \mu_{0}}{\pi \rho^{2 n}} \cdot \frac{e^{e^{a_{1}}}}{\left|a+a_{1}\right|^{k}\left|b+a_{1}\right|^{\ell}\left|c+a_{1}\right|^{m}}\right\},
$$

where $\mu_{0}=\pi(1+\gamma) /(1-\gamma)$ (cf. [1, Eqs. (2.28), (2.30), (2.34)]), or by (see von Sydow [12, Th. 1])

$$
\hat{e}_{n}(f)=\hat{e}_{n}^{\text {(Syd) }}(f)=\inf _{1<\rho<\rho_{\max }}\left\{\frac{4 \mu_{0}}{\left(1-\rho^{-2}\right) \rho^{2 n}} \cdot \frac{e^{e^{a_{1}}}}{\left|a+a_{1}\right|^{k}\left|b+a_{1}\right|^{\ell}\left|c+a_{1}\right|^{m}}\right\},
$$

or by (see Notaris [5, Eq. (3.28)])

$$
\begin{aligned}
\hat{e}_{n}(f)=\hat{e}_{n}^{(\mathrm{Not})}(f) & =\inf _{1<r<r_{\max }}\left\{\frac{2 \pi(1+\gamma)^{2} \tau^{2 n+1} r\left(\frac{r+1}{r-1}\right)^{1 / 2}}{\left(1-\gamma \tau^{2}\right)\left[1+\tau^{2 n+1}-\gamma \tau^{2}\left(1+\tau^{2 n-3}\right)\right]}\right. \\
& \left.\times \frac{e^{e^{r}}}{|a+r|^{k}|b+r|^{\ell}|c+r|^{m}}\right\},
\end{aligned}
$$

where $\tau=r-\sqrt{r^{2}-1}$ and $r_{\max }=|a|$.

Let the integrand $f$ be specialized by $k=1, \ell=5, m=10$, and

$$
\begin{gathered}
a=-1.202083333333333(+1), \quad b=-1.751428571428572(+1), \\
c=-2.301086956521739(+1),
\end{gathered}
$$

which means that $\rho_{\max }=24$ (numbers in parentheses indicate decimal exponents).

We have calculated the values of $\hat{e}_{n}^{(\mathrm{Sten})}(f), \hat{e}_{n}^{(\mathrm{Syd})}(f), \hat{e}_{n}^{(\mathrm{Not})}(f), e_{n}(f)$ for the corresponding integral $I(f)$ given by (3.1). The results show the effectiveness of the error bound (3.2) compared to the error bounds given by $\hat{e}_{n}^{(\mathrm{Sten})}(f), \hat{e}_{n}^{(\mathrm{Syd})}(f), \hat{e}_{n}^{(\mathrm{Not})}(f)$. For some values of $\gamma$ and $n=9,29$, the obtained results are displayed in Table 1,

In the end, let us consider numerical calculation of the integral (3.1), with

$$
f(t)=\bar{f}(t)=e^{e^{\cos (\omega t)}} \quad(\omega>0) .
$$

The function $\bar{f}(z)=e^{e^{\cos (\omega z)}}$ is entire, and it is easy to see that (see also [10])

$$
\max _{z \in C_{r}}\left|e^{e^{\cos (\omega z)}}\right|=e^{e^{\cosh (\omega r)}} \text { and } \max _{z \in \mathcal{E}_{\rho}}\left|e^{e^{\cos (\omega z)}}\right|=e^{e^{\cosh \left(\omega b_{1}\right)}},
$$

where $b_{1}=\frac{1}{2}\left(\rho-\rho^{-1}\right)$. The last maximums are attained on the imaginary axis.

For some values of $\gamma$, the obtained results of $\hat{e}_{29}^{(\text {Sten })}(\bar{f}), \hat{e}_{29}^{(\text {Syd })}(\bar{f}), \hat{e}_{29}^{(\text {Not })}(\bar{f}), e_{29}(\bar{f})$ are displayed in Table 2, for $\omega=0.1$ (left) and $\omega=1.2$ (right), and in Table 3, for $\omega=1.5$ (left) and $\omega=1.8$ (right). 
TABLE 1. The values of $\hat{e}_{n}^{(\text {Sten })}(f), \hat{e}_{n}^{(\text {Syd })}(f), \hat{e}_{n}^{(\text {Not })}(f), e_{n}(f)$ for some $\gamma \in(-1,0)$, in the cases $n=9$ (left) and $n=29$ (right).

\begin{tabular}{|l|l|l|l|l||l|l|l|l|}
\hline$\gamma$ & $\hat{e}_{9}^{(\text {Sten })}(f)$ & $\hat{e}_{9}^{(\mathrm{Syd})}(f)$ & $\hat{e}_{9}^{(\mathrm{Not})}(f)$ & $e_{9}(f)$ & $\hat{e}_{29}^{(\mathrm{Sten})}(f)$ & $\hat{e}_{29}^{(\mathrm{Syd})}(f)$ & $\hat{e}_{29}^{(\mathrm{Not})}(f)$ & $e_{29}(f)$ \\
\hline-0.99 & $3.74(-29)$ & $3.13(-29)$ & $2.28(-31)$ & $2.15(-31)$ & $2.80(-57)$ & $2.27(-57)$ & $1.51(-59)$ & $1.46(-59)$ \\
\hline-0.9 & $3.92(-28)$ & $3.28(-28)$ & $2.30(-29)$ & $2.17(-29)$ & $2.93(-56)$ & $2.37(-56)$ & $1.57(-57)$ & $1.47(-57)$ \\
\hline-0.8 & $8.27(-28)$ & $6.92(-28)$ & $9.32(-29)$ & $8.80(-29)$ & $6.19(-56)$ & $5.01(-56)$ & $6.09(-57)$ & $5.92(-57)$ \\
\hline-0.7 & $1.31(-27)$ & $1.10(-27)$ & $2.12(-28)$ & $2.00(-28)$ & $9.83(-56)$ & $7.96(-56)$ & $1.38(-56)$ & $1.34(-56)$ \\
\hline-0.6 & $1.86(-27)$ & $1.56(-27)$ & $3.81(-28)$ & $3.60(-28)$ & $1.39(-55)$ & $1.13(-55)$ & $2.47(-56)$ & $2.40(-56)$ \\
\hline-0.5 & $2.48(-27)$ & $2.07(-27)$ & $6.03(-28)$ & $5.69(-28)$ & $1.86(-55)$ & $1.50(-55)$ & $3.88(-56)$ & $3.76(-56)$ \\
\hline-0.4 & $3.19(-27)$ & $2.67(-27)$ & $8.78(-28)$ & $8.29(-28)$ & $2.39(-55)$ & $1.93(-55)$ & $5.61(-56)$ & $5.45(-56)$ \\
\hline-0.3 & $4.01(-27)$ & $3.35(-27)$ & $1.21(-27)$ & $1.14(-27)$ & $3.00(-55)$ & $2.43(-55)$ & $7.69(-56)$ & $7.47(-56)$ \\
\hline-0.2 & $4.96(-27)$ & $4.15(-27)$ & $1.60(-27)$ & $1.51(-27)$ & $3.72(-55)$ & $3.01(-55)$ & $1.01(-55)$ & $9.81(-56)$ \\
\hline-0.1 & $6.09(-27)$ & $5.09(-27)$ & $2.05(-27)$ & $1.93(-27)$ & $4.56(-55)$ & $3.69(-55)$ & $1.29(-55)$ & $1.25(-55)$ \\
\hline-0.01 & $7.29(-27)$ & $6.10(-27)$ & $2.50(-27)$ & $2.36(-27)$ & $5.46(-55)$ & $4.42(-55)$ & $1.56(-55)$ & $1.52(-55)$ \\
\hline
\end{tabular}

TABLE 2. The values of $\hat{e}_{29}^{(\mathrm{Sten})}(\bar{f}), \hat{e}_{29}^{(\mathrm{Syd})}(\bar{f}), \hat{e}_{29}^{(\mathrm{Not})}(\bar{f}), e_{29}(\bar{f})$ for some $\gamma \in(-1,0)$, in the cases $\omega=0.1$ (left) and $\omega=1.2$ (right).

\begin{tabular}{|l|l|l|l|l||l|l|l|l|}
\hline$\gamma$ & $\hat{e}_{29}^{(\text {Sten })}(\bar{f})$ & $\hat{e}_{29}^{(\mathrm{Syd})}(\bar{f})$ & $\hat{e}_{29}^{(\mathrm{Not})}(\bar{f})$ & $e_{29}(\bar{f})$ & $\hat{e}_{29}^{(\mathrm{Sten})}(\bar{f})$ & $\hat{e}_{29}^{(\mathrm{Syd})}(\bar{f})$ & $\hat{e}_{29}^{(\mathrm{Not})}(\bar{f})$ & $e_{29}(\bar{f})$ \\
\hline-0.99 & $1.85(-83)$ & $1.45(-83)$ & $8.43(-86)$ & $7.69(-86)$ & $8.70(-24)$ & $7.70(-24)$ & $7.60(-19)$ & $6.59(-26)$ \\
\hline-0.9 & $1.93(-82)$ & $1.52(-82)$ & $8.43(-84)$ & $7.56(-84)$ & $9.11(-23)$ & $8.07(-23)$ & $7.80(-17)$ & $5.66(-24)$ \\
\hline-0.8 & $4.08(-82)$ & $3.21(-82)$ & $3.37(-83)$ & $3.04(-83)$ & $1.93(-22)$ & $1.70(-22)$ & $3.21(-16)$ & $2.31(-23)$ \\
\hline-0.7 & $6.48(-82)$ & $5.09(-82)$ & $7.59(-83)$ & $7.04(-83)$ & $3.06(-22)$ & $2.71(-22)$ & $7.44(-16)$ & $5.31(-23)$ \\
\hline-0.6 & $9.18(-82)$ & $7.22(-82)$ & $1.35(-82)$ & $1.23(-82)$ & $4.33(-22)$ & $3.83(-22)$ & $1.36(-15)$ & $9.64(-23)$ \\
\hline-0.5 & $1.22(-81)$ & $9.62(-82)$ & $2.11(-82)$ & $1.96(-82)$ & $5.77(-22)$ & $5.11(-22)$ & $2.19(-15)$ & $1.54(-22)$ \\
\hline-0.4 & $1.57(-81)$ & $1.24(-81)$ & $3.04(-82)$ & $2.81(-82)$ & $7.42(-22)$ & $6.57(-22)$ & $3.26(-15)$ & $2.26(-22)$ \\
\hline-0.3 & $1.98(-81)$ & $1.55(-81)$ & $4.13(-82)$ & $3.82(-82)$ & $9.32(-22)$ & $8.26(-22)$ & $4.58(-15)$ & $3.15(-22)$ \\
\hline-0.2 & $2.45(-81)$ & $1.92(-81)$ & $5.40(-82)$ & $4.98(-82)$ & $1.15(-21)$ & $1.02(-21)$ & $6.17(-15)$ & $4.20(-22)$ \\
\hline-0.1 & $3.00(-81)$ & $2.36(-81)$ & $6.84(-82)$ & $6.26(-82)$ & $1.42(-21)$ & $1.25(-21)$ & $8.07(-15)$ & $5.44(-22)$ \\
\hline-0.01 & $3.60(-81)$ & $2.83(-81)$ & $8.27(-82)$ & $7.56(-82)$ & $1.70(-21)$ & $1.50(-21)$ & $1.01(-14)$ & $6.72(-22)$ \\
\hline
\end{tabular}

Observe that when the integrand, as in this case $\bar{f}$, becomes a more oscillating function, then the error bounds $\left.\hat{e}_{29}^{(\mathrm{Not})}(\bar{f})(\gamma \in(-1,0), \omega=1.2,1.5,1.8)\right)$, $e_{29}(\bar{f})(\gamma=-0.99,-0.9 ; \omega=1.5$ and $\gamma=-0.99,-0.9,-0.8 ; \omega=1.8)$ become much weaker than $\hat{e}_{29}^{(\text {Sten })}(\bar{f}), \hat{e}_{29}^{(\text {Syd })}(\bar{f})$. However, $e_{29}(\bar{f})$ remains as effective as $\hat{e}_{29}^{(\text {Sten })}(\bar{f}), \hat{e}_{29}^{(\text {Syd })}(\bar{f})$, for the values of $\gamma$, which are close 0 and larger values of $n\left(\rho^{*}\right.$ is close to 1). This confirms, for instance, the obtained results of $\hat{e}_{99}^{(\mathrm{Sten})}(\bar{f}), \hat{e}_{99}^{(\mathrm{Syd})}(\bar{f})$, $\hat{e}_{99}^{(\mathrm{Not})}(\bar{f}), e_{99}(\bar{f})$ for $\omega=2.2$, which are displayed in Table 4 . 
TABLE 3. The values of $\hat{e}_{29}^{(\mathrm{Sten})}(\bar{f}), \hat{e}_{29}^{(\mathrm{Syd})}(\bar{f}), \hat{e}_{29}^{(\mathrm{Not})}(\bar{f}), e_{29}(\bar{f})$ for some $\gamma \in(-1,0)$, in the cases $\omega=1.5$ (left) and $\omega=1.8$ (right).

\begin{tabular}{|l|l|l|l|l||l|l|l|l|}
\hline$\gamma$ & $\hat{e}_{29}^{(\text {Sten })}(\bar{f})$ & $\hat{e}_{29}^{(\mathrm{Syd})}(\bar{f})$ & $\hat{e}_{29}^{(\mathrm{Not})}(\bar{f})$ & $e_{29}(\bar{f})$ & $\hat{e}_{29}^{(\mathrm{Sten})}(\bar{f})$ & $\hat{e}_{29}^{(\mathrm{Syd})}(\bar{f})$ & $\hat{e}_{29}^{(\mathrm{Not})}(\bar{f})$ & $e_{29}(\bar{f})$ \\
\hline-0.99 & $1.61(-19)$ & $1.51(-19)$ & $8.35(-10)$ & $4.06(-10)$ & $2.24(-16)$ & $2.23(-16)$ & $9.81(+1)$ & $1.02(+120)$ \\
\hline-0.9 & $1.69(-18)$ & $1.59(-18)$ & $8.70(-08)$ & $4.75(-17)$ & $2.34(-15)$ & $2.34(-15)$ & $1.04(+4)$ & $1.62(+17)$ \\
\hline-0.8 & $3.57(-18)$ & $3.35(-18)$ & $3.65(-07)$ & $5.78(-19)$ & $4.95(-15)$ & $4.95(-15)$ & $4.49(+4)$ & $1.30(-09)$ \\
\hline-0.7 & $5.67(-18)$ & $5.31(-18)$ & $8.62(-07)$ & $1.08(-18)$ & $7.85(-15)$ & $7.84(-15)$ & $1.09(+5)$ & $5.68(-15)$ \\
\hline-0.6 & $8.03(-18)$ & $7.53(-18)$ & $1.61(-06)$ & $1.98(-18)$ & $1.11(-14)$ & $1.11(-14)$ & $2.09(+5)$ & $3.03(-15)$ \\
\hline-0.5 & $1.07(-17)$ & $1.00(-17)$ & $2.65(-06)$ & $3.19(-18)$ & $1.48(-14)$ & $1.48(-14)$ & $3.54(+5)$ & $4.92(-15)$ \\
\hline-0.4 & $1.38(-17)$ & $1.29(-17)$ & $4.02(-06)$ & $4.74(-18)$ & $1.91(-14)$ & $1.91(-14)$ & $5.54(+5)$ & $7.36(-15)$ \\
\hline-0.3 & $1.73(-17)$ & $1.62(-17)$ & $5.78(-06)$ & $6.65(-18)$ & $2.40(-14)$ & $2.39(-14)$ & $8.22(+5)$ & $1.04(-14)$ \\
\hline-0.2 & $2.14(-17)$ & $2.01(-17)$ & $7.98(-06)$ & $8.97(-18)$ & $2.97(-14)$ & $2.96(-14)$ & $1.18(+6)$ & $1.42(-14)$ \\
\hline-0.1 & $2.63(-17)$ & $2.46(-17)$ & $1.07(-05)$ & $1.17(-17)$ & $3.64(-14)$ & $3.64(-14)$ & $1.64(+6)$ & $1.87(-14)$ \\
\hline-0.01 & $3.15(-17)$ & $2.95(-17)$ & $1.36(-05)$ & $1.46(-17)$ & $4.36(-14)$ & $4.36(-14)$ & $2.17(+6)$ & $2.35(-14)$ \\
\hline
\end{tabular}

TABLE 4

\begin{tabular}{|c|c|c|c|c|}
\hline$\gamma$ & $\hat{e}_{99}^{(\text {Sten })}(\bar{f})$ & $\hat{e}_{99}^{(\text {Syd })}(\bar{f})$ & $\hat{e}_{99}^{(\text {Not) }}(\bar{f})$ & $e_{99}(\bar{f})$ \\
\hline-0.5 & $1.62(-54)$ & $1.64(-54)$ & $4.37(+34)$ & $5.48(-55)$ \\
\hline-0.4 & $2.09(-54)$ & $2.11(-54)$ & $7.00(+34)$ & $8.21(-55)$ \\
\hline-0.3 & $2.62(-54)$ & $2.65(-54)$ & $1.07(+35)$ & $1.16(-54)$ \\
\hline-0.2 & $3.25(-54)$ & $3.28(-54)$ & $1.57(+35)$ & $1.59(-54)$ \\
\hline-0.1 & $3.99(-54)$ & $4.03(-54)$ & $2.26(+35)$ & $2.10(-54)$ \\
\hline-0.01 & $4.78(-54)$ & $4.82(-54)$ & $3.08(+35)$ & $2.64(-54)$ \\
\hline
\end{tabular}

\section{Appendix}

We transform (2.4) to $(n \geq 2)$ and get

$$
\frac{\rho^{n+1 / 2}}{\sqrt{2} \pi(1+\gamma)^{2}}\left|K_{n, \gamma}^{(-1 / 2,1 / 2)}(z)\right|^{2}=\sqrt{\frac{E+E_{1}}{B+B_{1}}} \frac{A+A_{1}}{C+C_{1}} \cdot \sqrt{\frac{E+E_{1}}{B+B_{1}}} \frac{1}{D+D_{1}},
$$

and then looking for sufficient conditions ensuring that the $\sqrt{\frac{E+E_{1}}{B+B_{1}}} \frac{A+A_{1}}{C+C_{1}}$, namely $\sqrt{\frac{E+E_{1}}{B+B_{1}}} \frac{1}{D+D_{1}}$, attains its maximal value at $\theta=0$.

First, let us consider when $\sqrt{\frac{E+E_{1}}{B+B_{1}}} \frac{1}{D+D_{1}}$ attains its maximal value at $\theta=0$, i.e., when

$$
\sqrt{\frac{E+E_{1}}{B+B_{1}}} \frac{1}{D+D_{1}} \leq \sqrt{\frac{E}{B}} \frac{1}{D} .
$$

The last inequality holds if

$$
E D_{1}\left(B+B_{1}\right)\left[D+\left(D+D_{1}\right)\right]+D^{2}\left(E B_{1}-B E_{1}\right) \geq 0 .
$$


The last inequality holds trivially for $\theta=0$. After dividing by $2 \sin ^{2}(\theta / 2)(\theta \in(0, \pi])$ it reduces to the inequality which holds if

$$
\begin{aligned}
F_{d}(\rho) \equiv F_{d}(\rho ; n, \gamma) & =\left(a_{1}^{2}-1\right)\left(-(2 n+1)^{2}-\gamma^{2}(2 n-3)^{2}+2 \gamma a_{2}(2 n-1)^{2}\right) \\
& +2\left(a_{1}+1\right)\left(-1-\gamma^{2}+2 \gamma a_{2}\right)+8 \gamma a_{2 n-1}\left(a_{1}+1\right) \cdot h_{\max } \\
& +2 a_{1}\left(a_{2 n+1}+1+\gamma^{2}\left(a_{2 n-3}+1\right)-2 \gamma\left(a_{2 n-1}+a_{2}\right)\right) \geq 0 .
\end{aligned}
$$

We have used that, for the function

$$
h(\theta)=\cos ^{2} \frac{\theta}{2}\left(a_{1}-\cos \theta\right), \quad \theta \in[0, \pi],
$$

it can be easily shown that

$$
h_{\max }= \begin{cases}\frac{\left(a_{1}+1\right)^{2}}{8}, & 1<\rho \leq 3+2 \sqrt{2}, \\ a_{1}-1, & \rho>3+2 \sqrt{2} .\end{cases}
$$

Since $F_{d}(\rho)$ is continuous on $\mathbb{R}$ and $\lim _{\rho \rightarrow+\infty} F_{d}(\rho)=+\infty$ (when $n, \gamma$ are fixed), it follows that there exists $\rho_{d}^{*}$ such that for each $\rho>\rho_{d}^{*}$, where $\rho_{d}^{*}$ is the largest zero of $F_{d}(\rho), F_{d}(\rho)$ is positive. A typical graph of the function $F_{d}(\rho)$ [here for $n=2, \gamma=-0.99, \rho \in(1,3+2 \sqrt{2})]$ is displayed in Figure 5 (left).

For some values of $n$ and $\gamma \in(-1,0)$, the values of $\rho_{d}^{*}$ are displayed in Tables 5 and 6 .

TABLE 5

\begin{tabular}{|c|c|c|c|c|c|}
\hline$\rho_{d}^{*}$ & $\gamma=-0.99$ & $\gamma=-0.9$ & $\gamma=-0.8$ & $\gamma=-0.7$ & $\gamma=-0.6$ \\
\hline$n=2$ & 5.053 & 4.710 & 4.343 & 3.991 & 3.653 \\
\hline$n=3$ & 3.569 & 3.354 & 3.128 & 2.912 & 2.706 \\
\hline$n=9$ & 3.053 & 2.810 & 2.552 & 2.304 & 2.064 \\
\hline$n=29$ & 3.053 & 2.810 & 2.552 & 2.304 & 2.062 \\
\hline$n=49$ & 3.053 & 2.810 & 2.552 & 2.304 & 2.062 \\
\hline$n=99$ & 3.053 & 2.810 & 2.552 & 2.304 & 2.062 \\
\hline
\end{tabular}

TABLE 6

\begin{tabular}{|c|c|c|c|c|c|c|}
\hline$\rho_{d}^{*}$ & $\gamma=-0.5$ & $\gamma=-0.4$ & $\gamma=-0.3$ & $\gamma=-0.2$ & $\gamma=-0.1$ & $\gamma=-0.01$ \\
\hline$n=2$ & 3.328 & 3.018 & 2.721 & 2.438 & 2.166 & 1.926 \\
\hline$n=3$ & 2.508 & 2.317 & 2.132 & 1.952 & 1.775 & 1.613 \\
\hline$n=9$ & 1.833 & 1.624 & 1.462 & 1.350 & 1.265 & 1.199 \\
\hline$n=29$ & 1.825 & 1.588 & 1.344 & 1.137 & 1.086 & 1.061 \\
\hline$n=49$ & 1.825 & 1.588 & 1.344 & 1.097 & 1.052 & 1.036 \\
\hline$n=99$ & 1.825 & 1.588 & 1.344 & 1.081 & 1.026 & 1.018 \\
\hline
\end{tabular}


For some values of $\gamma(\in(-1,0)), n$, it is possible to obtain smaller values of $\rho_{d}^{*}$. Therefore, we are again interested in the answer to the question of when $\sqrt{\frac{E+E_{1}}{B+B_{1}}} \frac{1}{D+D_{1}}$ attains its maximal value at $\theta=0$, i.e., when $\sqrt{\left(E+E_{1}\right) /\left(B+B_{1}\right)} /$ $\left(D+D_{1}\right) \leq \sqrt{E / B} / D$. Since it holds that

$$
\sqrt{E+E_{1}}=\sqrt{a_{1}+\cos \theta} \leq \sqrt{a_{1}+1}, \quad \theta \in[0, \pi]
$$

and

$$
\begin{aligned}
\frac{1}{\sqrt{B+B_{1}}} & =\frac{1}{\sqrt{a_{1}-\cos \theta}} \leq \frac{1}{\frac{1}{\sqrt{2 \rho}} \sqrt{(\rho-\cos \theta)^{2}+\sin ^{2} \theta}} \\
& \leq \frac{1}{\frac{1}{\sqrt{2 \rho}}(\rho-\cos \theta)} \leq \frac{1}{\frac{1}{\sqrt{2 \rho}}(\rho-1)}=\frac{1}{\sqrt{a_{1}-1}}, \quad \theta \in[0, \pi],
\end{aligned}
$$

this question reduces to: When $1 /\left(\left(F+F_{1}\right)\left(D+D_{1}\right)\right)$, where $F=\rho-1(>0), F_{1}=$ $2 \sin ^{2}(\theta / 2), F+F_{1}=\rho-\cos \theta(>0)$ attains its maximal value at $\theta=0$, i.e., when

$$
\frac{1}{\left(F+F_{1}\right)\left(D+D_{1}\right)} \leq \frac{1}{F D} .
$$

The last inequality holds if

$$
\left(F+F_{1}\right) D_{1}+F_{1} D \geq 0 .
$$

The last inequality trivially holds for $\theta=0$. After dividing by $2 \sin ^{2}(\theta / 2), \theta \in(0, \pi]$ it reduces to the inequality, which holds if

$$
\begin{aligned}
\widetilde{F}_{d}(\rho) \equiv \widetilde{F}_{d}(\rho ; n, \gamma) & =(\rho-1)\left(-(2 n+1)^{2}-\gamma^{2}(2 n-3)^{2}+2 \gamma a_{2}(2 n-1)^{2}\right) \\
& +2\left(-1-\gamma^{2}+2 \gamma a_{2}\right)+8 \gamma a_{2 n-1} \cdot g_{\max } \\
& +a_{2 n+1}+1+\gamma^{2}\left(a_{2 n-3}+1\right)-2 \gamma\left(a_{2 n-1}+a_{2}\right) \geq 0 .
\end{aligned}
$$

We have used that, for the function

$$
g(\theta)=\cos ^{2} \frac{\theta}{2}(\rho-\cos \theta), \quad \theta \in[0, \pi],
$$

it can be easily shown that

$$
g_{\max }= \begin{cases}\frac{(\rho+1)^{2}}{8}, & 1<\rho \leq 3, \\ \rho-1, & \rho>3 .\end{cases}
$$

Since $\widetilde{F}_{d}(\rho)$ is continuous on $\mathbb{R}$ and $\lim _{\rho \rightarrow+\infty} \widetilde{F}_{d}(\rho)=+\infty$ (when $n, \gamma$ are fixed), it follows that there exists $\widetilde{\rho}_{d}^{*}$ such that for each $\rho>\widetilde{\rho}_{d}^{*}$, where $\widetilde{\rho}_{d}^{*}$ is the largest zero of $\widetilde{F}_{d}(\rho), \widetilde{F}_{d}(\rho)$ is positive. A typical graph of the function $\widetilde{F}_{d}(\rho)$ is analogous to that of $F_{d}(\rho)$.

For some values $n, \gamma(\gamma \in(-1,0))$ the values of $\widetilde{\rho}_{d}^{*}$ are displayed in Table 7. Some of them are smaller than the corresponding values of $\rho_{d}^{*}$. Because of that, we choose $\rho_{d}^{*}:=\min \left\{\rho_{d}^{*}, \widetilde{\rho}_{d}^{*}\right\}$.

Second, let us consider when $\sqrt{\frac{E+E_{1}}{B+B_{1}}} \frac{A+A_{1}}{C+C_{1}}$ attains its maximal value at $\theta=0$, i.e., when

$$
\sqrt{\frac{E+E_{1}}{B+B_{1}}} \frac{A+A_{1}}{C+C_{1}} \leq \sqrt{\frac{E}{B}} \frac{A}{C} .
$$


TABLE 7

\begin{tabular}{|c|c|c|c|c|c|c|}
\hline$\widetilde{\rho}_{d}^{*}$ & $\gamma=-0.5$ & $\gamma=-0.4$ & $\gamma=-0.3$ & $\gamma=-0.2$ & $\gamma=-0.1$ & $\gamma=-0.01$ \\
\hline$n=2$ & 4.843 & 4.146 & 3.532 & 3.025 & 2.632 & 2.356 \\
\hline$n=3$ & 3.186 & 2.777 & 2.480 & 2.250 & 2.062 & 1.919 \\
\hline$n=9$ & 1.622 & 1.515 & 1.455 & 1.410 & 1.371 & 1.340 \\
\hline$n=29$ & 1.185 & 1.163 & 1.150 & 1.140 & 1.130 & 1.122 \\
\hline$n=49$ & 1.109 & 1.100 & 1.093 & 1.088 & 1.082 & 1.078 \\
\hline$n=99$ & 1.056 & 1.053 & 1.050 & 1.047 & 1.044 & 1.042 \\
\hline
\end{tabular}

For $\theta \in[0, \pi / 2]$ it holds that

$$
\begin{aligned}
\sqrt{\frac{E+E_{1}}{B+B_{1}}} & =\frac{\sqrt{a_{1}+\cos \theta}}{\sqrt{a_{1}-\cos \theta}}=\sqrt{\frac{(\rho+\cos \theta)^{2}+\sin ^{2} \theta}{(\rho-\cos \theta)^{2}+\sin ^{2} \theta}} \leq \frac{\rho+\cos \theta}{\rho-\cos \theta} \\
& \leq \frac{\frac{1}{\sqrt{2 \rho}}(\rho+1)}{\frac{1}{\sqrt{2 \rho}}(\rho-1)}=\frac{\sqrt{a_{1}+1}}{\sqrt{a_{1}-1}},
\end{aligned}
$$

since

$$
\frac{(\rho+\cos \theta)^{2}+\sin ^{2} \theta}{(\rho-\cos \theta)^{2}+\sin ^{2} \theta} \leq \frac{(\rho+\cos \theta)^{2}}{(\rho-\cos \theta)^{2}}
$$

is fulfilled, due to the fact that

$(\rho+\cos \theta)^{2}(\rho-\cos \theta)^{2}+\sin ^{2} \theta(\rho+\cos \theta)^{2}-(\rho+\cos \theta)^{2}(\rho-\cos \theta)^{2}-\sin ^{2} \theta(\rho-\cos \theta)^{2} \geq 0$, i.e.,

$$
4 \rho \cos \theta \sin ^{2} \theta \geq 0, \quad \text { for } \quad \theta \in[0, \pi / 2] .
$$

For $\theta \in[\pi / 2, \pi], \sqrt{\frac{E+E_{1}}{B+B_{1}}} \frac{A+A_{1}}{C+C_{1}}$ attains its maximal value at $\theta=\pi / 2$, since $\cos \theta \leq 0$ on $[\pi / 2, \pi]$, and $\frac{A+A_{1}}{C+C_{1}}$ is a symmetric function on $[0, \pi]$ subject to $\theta=\pi / 2$ and, on $[0, \pi / 2]$, attains its maximum value at $\theta=\pi / 2$ (cf. [9, Lemma 2.1]).

We conclude that (4.1) holds, if the function

$$
\frac{\rho+\cos \theta}{\rho-\cos \theta} \cdot \frac{A+A_{1}}{C+C_{1}}
$$

attains its maximum on the interval $[0, \pi / 2]$ at $\theta=0$. This holds, if (by using the above notation and $\left.G=\rho+1(>0), G_{1}=-2 \sin ^{2}(\theta / 2), G+G_{1}=\rho+\cos \theta(\geq 0)\right)$

$$
\frac{G+G_{1}}{F+F_{1}} \cdot \frac{A+A_{1}}{C+C_{1}} \leq \frac{G A}{F C}
$$

i.e.,

$$
G A F C_{1}+G A F_{1}\left(C+C_{1}\right)-F C G A_{1}-F C G_{1}\left(A+A_{1}\right) \geq 0 .
$$

The last inequality trivially holds for $\theta=0$. After dividing by $2 \sin ^{2}(\theta / 2)(\theta \in$ $(0, \pi / 2])$ it reduces to the inequality, which by the substitution $\cos \theta=x(x \in[0,1))$ obtains the form

$$
\varphi(x) \geq 0
$$


where

$$
\begin{aligned}
\varphi(x) \equiv & \varphi_{\rho, \gamma}(x)=8 \gamma\left(\rho^{2}-1\right)\left(1-\gamma \rho^{2}\right)^{2}(1+x)\left(\left(1+\gamma^{2}\right) a_{2}-2 \gamma x^{2}\right) \\
+ & (\rho+1)\left(1-\gamma \rho^{2}\right)^{2}\left[\left(1+\gamma^{2}\right)^{2}-4 \gamma\left(1+\gamma^{2}\right) a_{2}\left(2 x^{2}-1\right)\right. \\
& \left.+2 \gamma^{2}\left(a_{4}+2\left(2 x^{2}-1\right)^{2}-1\right)\right] \\
- & 4 \gamma \rho^{2}\left(\rho^{2}-1\right)\left[\left(1+\gamma^{2}\right)^{2}-4 \gamma\left(1+\gamma^{2}\right) a_{2}+2 \gamma^{2}\left(a_{4}+1\right)\right](1+x) \\
+ & (\rho-1)\left[\left(1+\gamma^{2}\right)^{2}-4 \gamma\left(1+\gamma^{2}\right) a_{2}+2 \gamma^{2}\left(a_{4}+1\right)\right]\left(1+\gamma^{2} \rho^{4}-2 \gamma \rho^{2}\left(2 x^{2}-1\right)\right) .
\end{aligned}
$$

Further, we have

$$
\varphi^{\prime}(x)=\bar{a}_{3} x^{3}+\bar{a}_{2} x^{2}+\bar{a}_{1} x+\bar{a}_{0},
$$

where

$$
\begin{gathered}
\bar{a}_{3}=64 \gamma^{2}(\rho+1)\left(1-\gamma \rho^{2}\right)^{2}, \quad \bar{a}_{2}=-46 \gamma^{2}\left(\rho^{2}-1\right)\left(1-\gamma \rho^{2}\right)^{2}, \\
\bar{a}_{1}=-8 \gamma \rho^{2}(\rho-1)\left(\left(1+\gamma^{2}\right)^{2}-4 \gamma\left(1+\gamma^{2}\right) a_{2}+2 \gamma^{2}\left(a_{4}+1\right)\right) \\
-16 \gamma(\rho+1)\left(1-\gamma \rho^{2}\right)^{2}\left(2 \gamma \rho+\left(1+\gamma^{2}\right) a_{2}\right), \\
\bar{a}_{0}=4 \gamma\left(\rho^{2}-1\right)\left[2\left(1-\gamma \rho^{2}\right)^{2}\left(1+\gamma^{2}\right) a_{2}-\rho^{2}\left(\left(1+\gamma^{2}\right)^{2}\right.\right. \\
\left.\left.-4 \gamma\left(1+\gamma^{2}\right) a_{2}+2 \gamma^{2}\left(a_{4}+1\right)\right)\right],
\end{gathered}
$$

and

$$
\varphi^{\prime \prime}(x)=3 \bar{a}_{3} x^{2}+2 \bar{a}_{2} x+\bar{a}_{1} .
$$

Since $\bar{a}_{3}>0$, we conclude that the polynomial $\varphi^{\prime}(x)$ has at least one real zero $x_{0}$. In the case when the discriminant $D$ of $\varphi^{\prime \prime}(x)=0$ is negative, then $\varphi^{\prime}(x)$ strongly increases on $\mathbb{R}$, and $x_{0}$ is its unique real zero (in which $\varphi(x)$ attains its minimum). In that case we consider the function

$$
F_{l}(\rho) \equiv F_{l}(\rho, \gamma)= \begin{cases}\varphi(0), & x_{0}<0 \\ \varphi\left(x_{0}\right), & 0 \leq x_{0}<1 \\ \varphi(1), & 1 \leq x_{0}\end{cases}
$$

and conclude that (4.2) holds, if

$$
F_{l}(\rho) \geq 0 \text {. }
$$

Now, we are interested in the case when the discriminant $D$ of $\varphi^{\prime \prime}(x)=0$ is negative. We have $D=4 \bar{a}_{2}^{2}-12 \bar{a}_{3} \bar{a}_{1}=-4 \cdot 192 \gamma^{3}\left(1-\gamma \rho^{2}\right)^{2} \cdot D_{\gamma}(\rho)$, where

$$
\begin{aligned}
D_{\gamma}(\rho)= & -12 \gamma\left(\rho^{2}-1\right)^{2}\left(1-\gamma \rho^{2}\right)^{2}-16\left(1+\gamma^{2}\right) a_{2}(1+\rho)^{2}\left(1-\gamma \rho^{2}\right)^{2} \\
& -32 \gamma \rho(1+\rho)^{2}\left(1-\gamma \rho^{2}\right)^{2}-8 \gamma \rho^{2}\left(\rho^{2}-1\right)\left(\left(1+\gamma^{2}\right)^{2}-4 \gamma\left(1+\gamma^{2}\right) a_{2}\right. \\
& \left.+2 \gamma^{2}\left(a_{4}+1\right)\right) .
\end{aligned}
$$

Since $D_{\gamma}(\rho)$, therefore $D$, is continuous on $\mathbb{R}$ and $\lim _{\rho \rightarrow+\infty} D_{\gamma}(\rho)=-\infty$ (when $\gamma$ is fixed), it follows that there exists $\hat{\rho}=\hat{\rho}(\gamma)$ such that for each $\rho>\hat{\rho}$, where $\hat{\rho}$ is the largest zero of $D_{\gamma}(\rho), D_{\gamma}(\rho)$ is negative. Typical graphs of the functions $D_{\gamma}(\rho)$, for instance, for $\gamma=-0.99, \rho \in(1,1.8]$ (left) and for $\gamma=-0.7, \rho \in(1,1.8]$ (right) are displayed in Figure 2,

For some values $\gamma(\gamma \in(-1,0))$ the values of $\hat{\rho}$ are displayed in Table 8 .

For finding the zero $x_{0}$ of $\varphi^{\prime}(x)=0$, i.e., of

$$
x^{3}+a x^{2}+b x+c=0,
$$



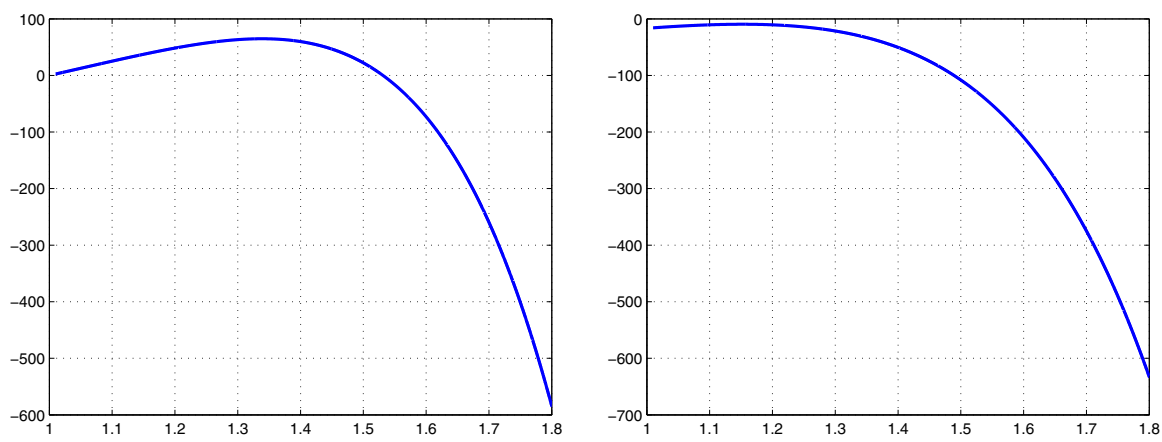

FiguRE 2. Typical graphs of the functions $D_{\gamma}(\rho)$ for $\gamma=$ $-0.99, \rho \in(1,1.8]$ (left) and for $\gamma=-0.7, \rho \in(1,1.8]$ (right).

TABLE 8

\begin{tabular}{|c|c|c|c|c|c|c|c|c|c|c|c|}
\hline$\gamma$ & -0.99 & -0.9 & -0.8 & -0.7 & -0.6 & -0.5 & -0.4 & -0.3 & -0.2 & -0.1 & -0.01 \\
\hline$\hat{\rho}$ & 1.532 & 1.484 & 1.371 & 1 & 1 & 1 & 1 & 1 & 1 & 1 & 1 \\
\hline
\end{tabular}

where $a=\bar{a}_{2} / \bar{a}_{3}, b=\bar{a}_{1} / \bar{a}_{3}, c=\bar{a}_{0} / \bar{a}_{3}$, we use the well-known Cardano's formula (see http://turnbull.mcs.st-and.ac.uk/ history/Mathematicians/Cardan.html or Wikipedia):

$$
x_{0}=\left[-\frac{q}{2}+\sqrt{\frac{q^{2}}{4}+\frac{p^{3}}{27}}\right]^{1 / 3}+\left[-\frac{q}{2}-\sqrt{\frac{q^{2}}{4}+\frac{p^{3}}{27}}\right]^{1 / 3}-\frac{a}{3},
$$

where

$$
p=b-a^{2} / 3, \quad q=2 a^{3} / 27-a b / 3+c .
$$

Since $F_{l}(\rho)$, in (4.3), is continuous on $\mathbb{R}$ and $\lim _{\rho \rightarrow+\infty} F_{l}(\rho)=+\infty$ (when $\gamma$ is fixed), it follows that there exists $\rho_{l}^{*}=\rho_{l}^{*}(\gamma)$ such that for each $\rho>\rho_{l}^{*}$, where $\rho_{l}^{*}$ is the largest zero of $F_{l}(\rho), F_{l}(\rho)$ is positive. We also take $\rho_{l}^{*}$ to be greater than or equal to the corresponding value of $\hat{\rho}$.

Let, for instance, $\gamma=-0.99, \rho \in[1.532,2.9]$. The function $q^{2} / 4+p^{3} / 27$ is positive (see Figure 3 (left)) and the square root of it is defined; the function $-q / 2+$ $\sqrt{q^{2} / 4+p^{3} / 27}$ is positive (see Figure 4 (left)), the function $-q / 2-\sqrt{q^{2} / 4+p^{3} / 27}$ is negative (see Figure 4 (right)). Therefore,

$$
x_{0}=\left[-\frac{q}{2}+\sqrt{\frac{q^{2}}{4}+\frac{p^{3}}{27}}\right]^{1 / 3}-\left[\frac{q}{2}+\sqrt{\frac{q^{2}}{4}+\frac{p^{3}}{27}}\right]^{1 / 3}-\frac{a}{3},
$$

and its graph (for $\gamma=-0.99, \rho \in[1.532,2.9]$ ) is displayed in Figure 3 (right). Observe that $x_{0} \in[0,1]$. The typical graph of the function $F_{l}(\rho)$ is displayed in Figure 5 (right). 

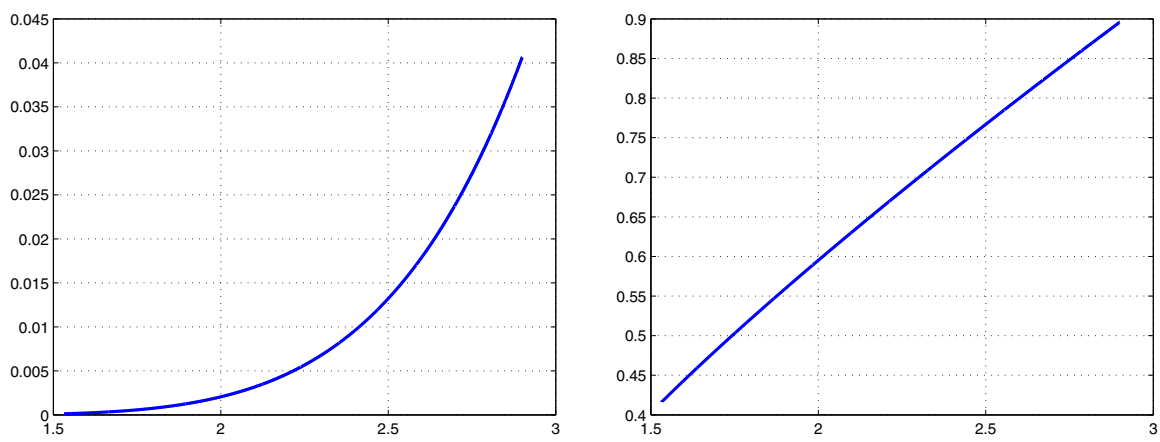

Figure 3. The functions $q^{2} / 4+p^{3} / 27$ for $\gamma=-0.99, \rho \in$ $[1.532,2.9]$ (left) and $x_{0}=x_{0}(\rho)$ for $\gamma=-0.99, \rho \in[1.532,2.9]$ (right).
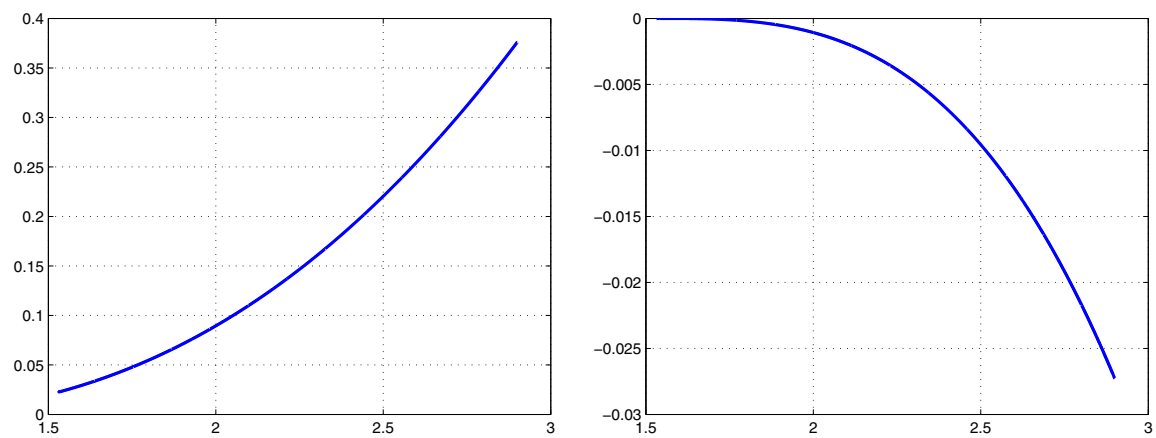

Figure 4 . The functions $-q / 2+\sqrt{q^{2} / 4+p^{3} / 27}$ (left) and $-q / 2-$ $\sqrt{q^{2} / 4+p^{3} / 27}$ (right) for $\gamma=-0.99, \rho \in[1.532,2.9]$.
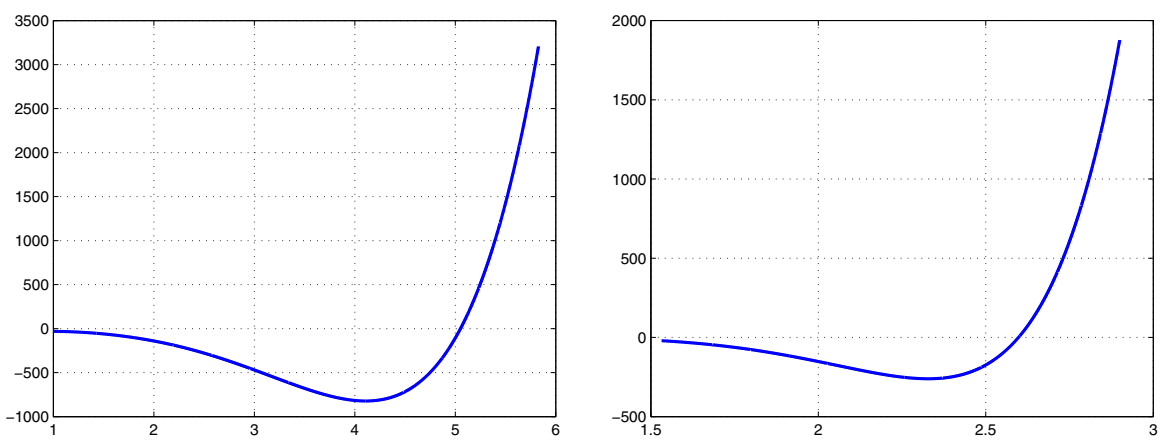

Figure 5. The typical graphs of the functions $F_{d}(\rho)$ (left) and $F_{l}(\rho)$ (right). 
For some values of $\gamma \in(-1,0)$, the values of $\rho_{l}^{*}$ are displayed in Table 9 .

Finally, we take for the values of $\rho^{*}$ in Theorem 2.1 to be $\rho^{*}=\max \left\{\rho_{l}^{*}, \rho_{d}^{*}\right\}$. For some values $n, \gamma(\gamma \in(-1,0))$ the values of $\rho^{*}$ are displayed in Tables 10 and 11 .

TABle 9

\begin{tabular}{|c|c|c|c|c|c|c|c|c|c|c|c|}
\hline$\gamma$ & -0.99 & -0.9 & -0.8 & -0.7 & -0.6 & -0.5 & -0.4 & -0.3 & -0.2 & -0.1 & -0.01 \\
\hline$\rho_{l}^{*}$ & 2.598 & 2.089 & 1.571 & 1 & 1 & 1 & 1 & 1 & 1 & 1 & 1 \\
\hline
\end{tabular}

TABLE 10

\begin{tabular}{|c|c|c|c|c|c|}
\hline$\rho^{*}$ & $\gamma=-0.99$ & $\gamma=-0.9$ & $\gamma=-0.8$ & $\gamma=-0.7$ & $\gamma=-0.6$ \\
\hline$n=2$ & 5.053 & 4.710 & 4.343 & 3.991 & 3.653 \\
\hline$n=3$ & 3.569 & 3.354 & 3.128 & 2.912 & 2.706 \\
\hline$n=9$ & 3.053 & 2.810 & 2.552 & 2.304 & 2.064 \\
\hline$n=29$ & 3.053 & 2.810 & 2.552 & 2.304 & 2.062 \\
\hline$n=49$ & 3.053 & 2.810 & 2.552 & 2.304 & 2.062 \\
\hline$n=99$ & 3.053 & 2.810 & 2.552 & 2.304 & 2.062 \\
\hline
\end{tabular}

TABLE 11

\begin{tabular}{|c|c|c|c|c|c|c|}
\hline$\rho^{*}$ & $\gamma=-0.5$ & $\gamma=-0.4$ & $\gamma=-0.3$ & $\gamma=-0.2$ & $\gamma=-0.1$ & $\gamma=-0.01$ \\
\hline$n=2$ & 3.328 & 3.018 & 2.721 & 2.438 & 2.166 & 1.926 \\
\hline$n=3$ & 2.508 & 2.317 & 2.132 & 1.952 & 1.775 & 1.613 \\
\hline$n=9$ & 1.622 & 1.515 & 1.455 & 1.350 & 1.265 & 1.199 \\
\hline$n=29$ & 1.185 & 1.163 & 1.150 & 1.137 & 1.086 & 1.061 \\
\hline$n=49$ & 1.109 & 1.100 & 1.093 & 1.088 & 1.052 & 1.036 \\
\hline$n=99$ & 1.056 & 1.053 & 1.050 & 1.047 & 1.027 & 1.018 \\
\hline
\end{tabular}

\section{ACKNOWLEDGMENTS}

The author thanks the referee for helpful comments concerning this paper.

\section{REFERENCES}

1. W. Gautschi and S. E. Notaris, Gauss-Kronrod quadrature formulae for weight function of Bernstein-Szegö type, J. Comput. Appl. Math. 25 (1989), 199-224; erratum in J. Comput. Appl. Math. 27 (1989), 429. MR988057 (90d:65045)

2. W. Gautschi and R. S. Varga, Error bounds for Gaussian quadrature of analytic functions, SIAM J. Numer. Anal. 20 (1983), 1170-1186. MR723834 (85j:65010)

3. W. Gautschi, E. Tychopoulos and R. S. Varga, A note on the contour integral representation of the remainder term for a Gauss-Chebyshev quadrature rule, SIAM J. Numer. Anal. 27 (1990), 219-224. MR1034931 (91d:65044)

4. D. B. Hunter, Some error expansions for Gaussian quadrature, BIT 35 (1995), 64-82. MR1429008 (97i:65040) 
5. S. E. Notaris, The error norm of Gaussian quadrature formulae for weight functions of Bernstein-Szegő type, Numer. Math. 57 (1990), 271-283. MR1057125 (92a:65080)

6. F. Peherstorfer, On the remainder of Gaussian quadrature formulas for Bernstein-Szegö weight functions, Math. Comp. 60 (1993), 317-325. MR.1153169 (93d:65030)

7. R. Scherer, T. Schira, Estimating quadrature errors for analytic functions using kernel representations and biorthogonal systems, Numer. Math. 84 (2000), 497-518. MR.1738478 (2001d:65034)

8. T. Schira, The remainder term for analytic functions of symmetric Gaussian quadratures, Math. Comp. 66 (1997), 297-310. MR.1372009 (97c:65050)

9. M. M. Spalević and M. S. Pranić, Error bounds of certain Gaussian quadrature formulae, J. Comput. Appl. Math. 234 (2010), 1049-1057. MR2609559 (2011b:41047)

10. M. M. Spalević, M. S. Pranić and A. V. Pejčev, Maximum of the modulus of kernels of Gaussian quadrature formulae for one class of Bernstein-Szegő weight functions, Appl. Math. Comput. 218 (2012), 5746-5756.

11. F. Stenger, Bounds on the error of Gauss-type quadratures, Numer. Math. 8 (1966), 150-160. MR0196936(33:5120)

12. B. von Sydow, Error estimates for Gaussian quadrature formulae, Numer. Math. 29 (1977), 59-64. MR0471270 (57:11007)

Department of Mathematics, University of Beograd, Faculty of Mechanical Engineering, Kraljice Marije 16, 11120 Belgrade 35, Serbia

E-mail address: mspalevic@mas.bg.ac.rs 